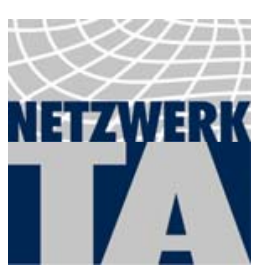

\section{Das Netzwerk TA - Profil und Aktivitäten}

Am 24. November 2004 wurde in Berlin das „Netzwerk TA“ gegründet. Gründungsmitglieder sind eine Reihe anerkannter Kolleginnen und Kollegen aus den unterschiedlichsten Arbeitsgebieten und Ausrichtungen der TA (siehe Kasten). Angesprochen sind Politik-, Wirtschafts- und Gesellschaftsberatung, also die praktische Seite der TA, ebenso wie die einschlägigen akademischen Disziplinen. An der konstituierenden Sitzung nahmen ca. 80 Forscher und Praktiker teil. Die in der dortigen Diskussion und auf der nachfolgenden 1. Konferenz des Netzwerks (,Technik in einer fragilen Welt") gegebenen Anregungen lassen sich zu folgendem Profil für das Netzwerk TA zusammenfügen.

\section{Hintergrund}

Wissenschaft, Technik und Innovation sind wesentliche Antriebskräfte der gesellschaftlichen Erneuerung und unerlässlich für Wohlstand, Sicherheit, Gesundheit und Lebensqualität. Gleichzeitig stellen sich neue Herausforderungen an Politik, Wirtschaft und Öffentlichkeit, die sich aus der wissenschaftlich-technischen und der damit verschränkten sozioökonomischen Entwicklung ergeben. Zu nennen wären hier u.a.: die Anforderungen einer nachhaltigen Entwicklung, die Zunahme des Innovationstempos in vielen Bereichen, die Sicherung der Wettbewerbsfähigkeit von Wirtschaft und Bildungssystem, ethisch relevante Herausforderungen traditioneller Menschenbilder und Gesellschaftsentwürfe, Fragen der Globalisierung, Umwälzungen in Lebens- und Arbeitswelt, neue Mechanismen der gesellschaftlichen Meinungsbildung und Entscheidungsfindung und neue Formen gesellschaftlicher Regulation (governance) sowie neue Wege in Wissensproduktion und Wissensverteilung.

\section{TA (Technikfolgenabschätzung/Technology} Assessment)

Aufgabe der TA ist es, durch vorausschauende Analyse von Chancen und Risiken von Wissenschaft und Technik die oben genannten Herausforderungen, ihre Voraussetzungen und Folgen zu erforschen, sie mit Hilfe wissenschaftlicher Methoden und nachvollziehbarer Kriterien zu bewerten und Problemlösungen bzw. Optionen zu erarbeiten, um Politik, Wirtschaft und Öffentlichkeit zu beraten und gesellschaftliche Meinungsbildungs- und Entscheidungsprozesse $\mathrm{zu}$ unterstützen. Diese Unterstützung bezieht sich sowohl auf die Informationsbasis als auch auf die zugrunde gelegten normativen Orientierungen. Sie berücksichtigt, dass Wissen und Werte in Fragen der Zukunftsgestaltung häufig nicht strikt zu trennen sind, und dass der Umgang mit Nichtwissen und Unsicherheiten eine wesentliche Herausforderung darstellt. Da Forschung und Beratung häufig miteinander verbunden sind, spielt auch die Kommunikation der Forschungsergebnisse, für den jeweiligen Adressaten optimiert, eine wichtige Rolle.

Die Art und Weise, wie diese Aufgaben bewältigt werden, unterscheidet sich in Abhängigkeit vom jeweiligen Kontext erheblich. Relevante Faktoren hierfür sind die spezifische TA-Fragestellung, der Adressat, die institutionelle Konstellation, der betreffende Wissenschafts- und Technikbezug, die gesellschaftliche Konfliktlage und die Akteurs- und Beteiligungsverhältnisse sowie die Kosten von Veränderungen. Diese Kontextabhängigkeit führt zu einer großen institutionellen, konzeptionellen und methodischen Diversität der TA.

\section{Das Netzwerk TA}

Das „Netzwerk TA“ ist ein Zusammenschluss von Wissenschaftlern, Experten und Praktikern im breit verstandenen Themenfeld TA. Dieser Kreis setzt sich zusammen aus den (teils überlappenden) Bereichen Technikfolgenabschätzung, Praktische Ethik, Systemanalyse, Risikoforschung, Technikgestaltung für nachhaltige Entwicklung, Innovations-, Institutionen- und Technikanalyse, Innovations- und Zukunftsforschung und den dabei involvierten wissenschaftlichen Disziplinen aus Natur-, Technik-, Wirtschafts- und Sozialwissenschaften, den 


\section{Gründungsmitglieder des „Netzwerk TA“}

Dr. Sergio Bellucci (Zentrum für Technologiefolgen-Abschätzung TA-SWISS, Bern)

Professor Dr. Raimund Bleischwitz (Wuppertal Institut für Klima, Umwelt, Energie GmbH)

Professor Dr. Alfons Bora (Institut für Wissenschafts- und Technikforschung, Universität Bielefeld)

Klaus Burmeister (Z-Punkt, The Foresight Company, Essen)

Dr. Peter Dienel (Zentrum Technik und Gesellschaft (ZTG) der Technischen Universität Berlin)

Professor Dr. Carl Friedrich Gethmann (Europäische Akademie zur Erforschung von Folgen wissenschaftlichtechnischer Entwicklungen Bad Neuenahr-Ahrweiler GmbH)

Professor Dr. Armin Grunwald (Institut für Technikfolgenabschätzung und Systemanalyse des Forschungszentrums Karlsruhe ITAS/FZK; Büro für Technikfolgenabschätzung am deutschen Bundestag (TAB), Berlin)

Professor Dr. Hartmut Hirsch-Kreinsen (Lehrstuhl Wirtschafts- und Industriesoziologie Universität Dortmund)

Professor Dr. Christoph Hubig (Institut für Philosophie Universität Stuttgart)

Professor Dr. Regine Kollek (FG Technologiefolgenabschätzung der modernen Biotechnologie in der Medizin/ Neurobiologie Universität Hamburg)

Professor Dr. Rolf Kreibich (Institut für Zukunftsstudien und Technologiebewertung, Berlin)

Professor Dr. Wolfgang Krohn (Institut für Wissenschafts- und Technikforschung, Universität Bielefeld)

Professor Dr. Frieder Mayer-Krahmer (Institut Systemtechnik und Innovationsforschung ISI Karlsruhe)

Dr. Walter Peissl (Institut für Technikfolgen-Abschätzung (ITA) der Österreichischen Akademie der Wissenschaften Wien)

Professor Dr. Ortwin Renn (Abteilung für Technik- und Umweltsoziologie des Instituts für Sozialwissenschaften, Universität Stuttgart)

Dr. Saykowski (BAYER AG)

Professor Dr. Georg Simonis (Lehrgebiet „Internationale Politik / Vergleichende Politikwissenschaft“ Fernuniversität Hagen)

Professor Dr. Arnim von Gleich (Fachgebiet Technikgestaltung und Technologieentwicklung Universität Bremen)

Dr. Dr. Axel Zweck (Zukünftige Technologien Consulting (ZTC), VDI-Technologie Zentrum)

Politik- und Rechtswissenschaften sowie der Philosophie.

Die Mitglieder des Netzwerks vertreten die verschiedenen Ausprägungen der TA und decken das weite Spektrum zwischen Theorie und Praxis, zwischen Forschung und Beratung sowie zwischen den verschiedenen wissenschaftlichen Disziplinen ab. Sie verstehen die dadurch entstehende Vielfalt als Chance, themenbezogen Kompetenzen und Erfahrungen zu bündeln und auf diese Weise zu einer optimalen Nutzung der Ressourcen beizutragen.

\section{Ziele und Aufgaben}

Das Netzwerk TA ist entstanden vor dem Hintergrund einer bislang offensichtlich unzureichenden Kooperation innerhalb der TA. Ziele und Aufgaben des Netzwerks sind
- Verbesserung der Kommunikation und des Informationsaustauschs innerhalb der TACommunity, zunächst im deutschsprachigen Raum, in der weiteren Perspektive auch darüber hinaus,

- Identifikation neuer Themen und Beratungsaufgaben,

- Initiierung und Durchführung selbst definierter Forschungsprojekte,

- systematische und kooperative Weiterentwicklung von TA-Konzepten und Methoden,

- Erarbeitung von Qualitätskriterien der TA und von Ansätzen der internen Qualitätssicherung,

- Formierung einer nach außen stärker sichtbaren „TA-Community“, bestehend aus den genannten Forschungs- und Beratungslinien,

- Förderung des wissenschaftlichen Nachwuchses, 
- Stärkung des Stellenwertes der TA in Wissenschaft und Gesellschaft,

- Entwicklung systematischer und effektiver Methoden der Umsetzung und gesellschaftspolitischen Nutzung von TA-Wissen.

\section{Aktivitäten}

Um diese Ziele zu erreichen, unternimmt das Netzwerk TA (in der ersten Phase) folgende konkreten Schritte:

- Einrichtung einer E-Mail-Liste und eines Internetportals für das Netzwerk mit Links zu den beteiligten Institutionen, einer News- und Ankündigungsseite, unter Nutzung bereits bestehender Angebote (z. B. die Web-Angebote des TA-NET NRW oder von ITAS),

- regelmäßige Berichterstattung über die Aktivitäten des „Netzwerks TA“ in der Zeitschrift „Technikfolgenabschätzung - Theorie und Praxis“,

- Durchführung von Workshops und Konferenzen,

- Durchführung eines jährlichen Netzwerktreffens in Verbindung mit Workshops oder Konferenzen des „Netzwerks TA“,

- Einrichtung von thematischen Arbeitskreisen.

Die erste Konferenz des Netzwerks TA (NTA1) hat vom 24. - 26. November 2004 unter dem Thema „Technik in einer fragilen Welt: Die Rolle der Technikfolgenabschätzung“ stattgefunden. Konferenzberichte sind $\mathrm{u}$. a. zu finden im „TAB-Brief“, Heft 2/2004 und in „Technikfolgenabschätzung. Theorie und Praxis“, Heft 3/2004. Eine Buchpublikation mit den Beiträgen der Konferenz in der ITAS-Schriftenreihe „Gesellschaft - Technik - Umwelt“ in der edition sigma ist in Vorbereitung.

(Michael Decker)

》)

\section{Aufbau des Netzwerks TA}

\section{Arbeitsgruppe luK des Netzwerks TA}

Als erste Arbeitsgruppe des Netzwerks TA hat sich die AG-IuK gebildet. Sie besteht zurzeit aus Katharina Drechsler (Universität Bielefeld), Ulrich Fiedeler (ITAS, Karlsruhe), Michael Nentwich (ITA, Wien) und Ulrich Riehm (ITAS, Karlsruhe). Sie hat sich zur Aufgabe gestellt, die informationstechnische Infrastruktur des NTA aufzubauen und zu pflegen. Die beiden ersten Aktivitäten waren die Einrichtung und die Betreuung einer Mailliste sowie der Aufbau eines Web-Servers für das Netzwerk TA. Ansprechpartner: Ulrich Riehm

\section{NTA-Mailingliste des Netzwerkes TA}

Die NTA-Mailingliste wurde am 17.12.2004 eingerichtet. In ihr sind (mit Stand Ende Februar) über 150 Teilnehmerinnen und Teilnehmer eingeschrieben. Über diese Mailingliste werden die Informationen von Netzwerkpartnern an alle Netzwerkpartner weitergeleitet. Die Inhalte der NTA-Mailingliste sind potenziell alle TArelevanten Themen wie Berichte über Forschungsprojekte und -resultate, Hinweise auf Ausschreibungen, Konferenzhinweise, Call for Papers, Stellenausschreibungen sowie natürlich die Aktivitäten des Netzwerks TA im engeren Sinne, wie thematische Arbeitsgruppen und Workshops. Zudem ist sie als Diskussionsforum gedacht, um über Erfahrungen mit und theoretische Konzepte der TA zu diskutieren. Sowohl von der Informations- wie von der Diskussionsfunktion wird bisher rege Gebrauch gemacht. Die NTA-Mailingliste ist zunächst bewusst als offene Liste konzipiert. Jeder TAInteressierte kann sich daran beteiligen, auch wenn er nicht formelles Mitglied im Netzwerk TA ist. Jeder angemeldete Teilnehmer an der NTA-Mailingliste kann ohne Einschaltung eines Moderators seine Mitteilungen direkt an alle übrigen Teilnehmer verschicken. Der Listen-Moderator, Ulrich Fiedeler von ITAS, wird lediglich auf die Einhaltung bestimmter Grundregeln achten, die Liste möglichst vor „Störern“ (Spam) schützen und für technische Fragen zur Verfügung stehen.

Wenn auch Sie sich bei der Mailingliste anmelden wollen, senden Sie eine E-Mail an 
LISTSERV@LISTSERV.DFN.DE mit dem Befehl „SUBSCRIBE NTA“ als einziger Inhalt des Mailtextes. Bei erfolgreicher Subskription erhalten Sie eine automatisch generierte Bestätigung, in der auch weitere Informationen zum Gebrauch der Mailingliste enthalten sind. Sollten Probleme bei der Anmeldung oder in der Nutzung auftreten. dann wenden Sie sich bitte an NTA-REQUEST@LISTSERV.DFN.DE.

\section{NTA-Web-Server online}

Unter der Adresse www.netzwerk-ta.net ist der Web-Server des Netzwerks TA seit Anfang März 2005 online. Er steht im Rechenzentrum der Universität Bielefeld und wird dort von Frau Drechsler betreut. Für Inhalt und Gestaltung des Web-Servers ist die Arbeitsgruppe IuK in Absprache mit dem Koordinationsteam des Netzwerks TA zuständig. Der NTA-Web-Server umfasst in seiner ersten Ausbaustufe einen Teil, der sich direkt auf das Netzwerk bezieht mit Informationen über die Ziele und Aufgaben des NTA, seine derzeitigen Gremien, sowie der Möglichkeit, sich online als persönliches oder institutionelles Mitglied im Netzwerk anzumelden. Der zweite Block berichtet über Aktivitäten des NTA, so über die Mailliste und Veranstaltungen. Der dritte Block bietet TA-relevante weiterführende Informationen mit zurzeit den folgenden Kategorien: TA-Kalender, TA-Links, TA-Zeitschriften und TA-Ausschreibungen.

\section{Kontakt}

Ansprechpartner für das Koordinationsteam:

Dr. Michael Decker

Forschungszentrum Karlsruhe

Institut für Technikfolgenabschätzung und Systemanalyse (ITAS)

Postfach 3640, D-76021 Karlsruhe

Tel.: 07247/82-3007 oder -2501 (Sekretariat)

Fax: 07247/82-4806

E-Mail: NetzwerkTA@itas.fzk.de

\section{Mitgliedschaft}

Online über das Anmeldeformular unter der WebAdresse

http://www.netzwerk-ta.net 